\title{
CD16/IL15/CD33 Trispecific Killer Cell Engager
}

National Cancer Institute

\section{Source}

National Cancer Institute. CD16/IL15/CD33 Trispecific Killer Cell Engager. NCI Thesaurus. Code C151948.

A trispecific killer eng ager (T riKE) molecule containing an anti-cluster of differentiation 16 (CD16; FcgammaRIII) single-chain variable fragment (scFv) to eng age natural killer (NK) cells, an anti-CD33 scFv to engage myeloid cells and a human modified interleukin-15 (IL15) linker, that links the two scFv, with potential immunomodulating and antineoplastic activities against CD33-expressing tumor cells. Upon administration of the CD16/IL15/CD33 TriKE, the simultaneous binding to CD16 on NK cells and CD33 on tumor cells will induce NK cell cytotoxicity specifically against CD33-expressing tumor cells. The cytokine IL-15 linker promotes NK cell proliferation, activity, survival and expansion. CD33 is expressed on normal non-pluripotent hematopoietic stem cells and is overexpressed on myeloid leukemia cells. 